\title{
Joint Relaying and Network Coding in Wireless Networks
}

\author{
Sachin Katti Ivana Marić Andrea Goldsmith Dina Katabi Muriel Médard

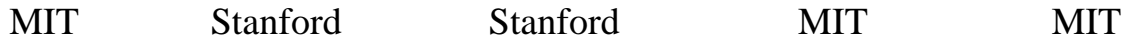

\begin{abstract}
Relaying is a fundamental building block of wireless networks. Sophisticated relaying strategies at the physical layer have been developed for a single flow, but multiple flows are typically handled by time sharing the channel between the flows at the network level. In this paper, time-sharing when forwarding two data streams at the relay is compared to joint relaying and network coding that allows the relay to combine data streams. Two commonly occurring blocks in wireless networks with both unicast and multicast traffic are considered. It is shown that joint relaying and network coding can achieve gains and even double the throughput for certain channel conditions.
\end{abstract}

\section{INTRODUCTION}

Wireless networks contain nodes that act as sources, destinations and relays and can communicate in variety of ways. Information-theoretic models of wireless networks allow for any encoding scheme that satisfies node constraints. Determining the optimum communication schemes in such general setting seems as a distant goal.

When there are multiple sources in a network, a relay will often need to handle multiple streams. Two different approaches have a relay time share in sending multiple data streams or, send combined information. The goal of this paper is to compare this two opposite directions in handling multiple streams.

The relay channel [6] is one of the building blocks of multihop wireless networks. Starting with fundamental coding strategies and outer bounds developed in [3], relaying has been extensively studied (see [4] and references therein). Because the relay node helps in transmitting data for a single source-destination pair, relay channel models do not capture cooperation in the case of multiple data sources. As a building block of larger networks, the relay channel thus implies relaying on the network layer, i.e. the relay time-shares in relaying different data streams.

This paper presents the benefits of simple analog network coding, i.e. network coding of physical signals carrying information from multiple sources, in the wireless channel. Two scenarios that are common building blocks of larger networks with unicast or multicast traffic are studied. We show that joint relaying and network coding achieves higher data rates when compared to routing at the relay. Furthermore, while the general case of relaying of multiple sources is considerably

\footnotetext{
${ }^{1}$ The work by I. Marić was supported from the DARPA ITMANET program under grant 1105741-1-TFIND and from the ARO under MURI award W911NF-05-1-0246
}

hard to analyze [1], [4], [5], [8], the achievable rates of analog network coding are relatively easy to evaluate.

In the first part of this paper, we consider the two-way relay channel [10] in which two nodes send messages to each other with a help of a relay node (see Fig. 1). Two transmitted signals interfere at the relay. We compare the performance of analog network coding to the pure routing approach in which relay time shares in relaying messages sent by nodes 1 and 2 . For this channel, achievable rate regions for three cooperative strategies were derived and compared in [7]. The considered strategies are extensions of decode-and-forward, compressand-forward and amplify-and-forward to the multiple source scenario. In fact, the joint encoding strategy we consider is the amplify-and-forward relaying scheme that was evaluated for the full-duplex case in [7]. We revisit this strategy with a different goal: we want to compare performance of such joint network coding and relaying approach to pure routing on the relay in order to demonstrate its gains and motivate such approach in future wireless networks. We perform the analysis for both the half-duplex and the full-duplex case.

We further extend the notion of joint relaying and network coding to the case of multicast traffic. Our analysis shows that this joint approach achieves gains in comparison to traditional relaying and time-sharing, both for unicast and multicast. We observe significant benefits from such marriage even with simple strategies. It is to be seen whether more complex joint relaying and network coding strategies can bring higher gains.

The rest of the paper is organized as follows. In Section II analog network coding is compared to the outer bounds on the routing performance in the two-way relay channel. This approach is then extended to the multicast relay channel in Section III. We conclude in Section IV.

\section{JOINT RELAYING AND NETWORK CODING FOR UNICAST}

We wish to compare joint relaying and network coding to traditional relaying, which we refer to as the routing approach. Towards that goal, we compare rates achievable with our scheme to outer bounds of traditional relaying.

We consider the two-way relay channel in Fig. 1. Nodes 1 and 2 wish to send their messages in $N$ channel uses to each other. We denote the rate from terminal $t$ as $R_{t}$. Encoders 1 and 2 transmit independent channel inputs $X_{1}$ and $X_{2}$, respectively and the relay transmits $X_{3}$. The received signal at the relay is

$$
Y_{3}=h_{13} X_{1}+h_{23} X_{2}+Z_{3}
$$




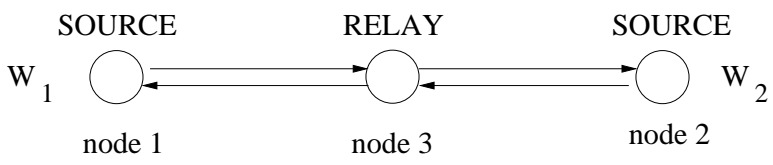

Fig. 1-Two-way relay channel.

and at the nodes 1 and 2

$$
\begin{aligned}
& Y_{1}=h_{21} X_{2}+h_{31} X_{3}+Z_{1} \\
& Y_{2}=h_{12} X_{1}+h_{32} X_{3}+Z_{2}
\end{aligned}
$$

where $Z_{t}$ are independent, zero-mean unit-variance Gaussian random variables. For simplicity, in this section we assume that nodes transmit with the same power $P$. We first describe the half-duplex scenario and then the full-duplex scenario.

\section{A. Half-Duplex Relaying}

\section{Routing: The Outer Bound}

We next compute the outer bound for traditional routing. The relay receives in one time slot and transmits in the next. Senders can transmit in both slots. The channel is time-shared between the two streams. We upper bound the capacity of the relay network using the cutset bound [3]. The capacity from node 1 to node 2 is upper-bounded by $\min \left\{C_{11}, C_{12}\right\}$, where

$$
\begin{aligned}
C_{11} & =\arg \max _{0 \leq \rho<1} \frac{1}{4} \log \left(1+\left(h_{12}^{2}+h_{31}^{2}\right) P\right) \\
& +\frac{1}{4} \log \left(1+\left(1-\rho^{2}\right) h_{12}^{2} P\right) \\
C_{12} & =\arg \max _{0 \leq \rho<1} \frac{1}{4} \log \left(1+\left(h_{12}^{2}+h_{32}^{2}\right) P\right) \\
& \left.+2 \rho P \sqrt{h_{12}^{2} h_{32}^{2}}\right)+\frac{1}{4} \log \left(1+h_{12}^{2} P\right) .
\end{aligned}
$$

Similar bounds exist for $R_{2}$, with $h_{31}$ and $h_{32}$ interchanged. It is assumed that each flow is relayed for the same amount of time, which could be optimized for higher rate.

\section{Network Coding: The Inner Bound}

We assume that nodes 1 and 2 simultaneously send in one time slot and the relay transmits in the next. Since nodes cannot transmit and receive at the same time, the direct channel between nodes 1 and 2 cannot be exploited. The relay amplifies the received signal and broadcasts it. We have

$$
X_{3}[n]=\alpha Y_{3}[n]
$$

where amplification factor $\alpha$ is chosen such that the relay power constraint is satisfied

$$
\alpha \leq \sqrt{\frac{P_{3}}{h_{13}^{2} P_{1}+h_{23}^{2} P_{2}+1}} .
$$

The signal (2) at node 1 becomes

$$
\begin{aligned}
Y_{1}[n] & =h_{31} \alpha Y_{3}[n]+Z_{1}[n] \\
& =\alpha h_{31}\left(h_{13} X_{1}[n]+h_{23} X_{2}[n]\right) \\
& +\alpha h_{31} Z_{3}[n]+Z_{1}[n] .
\end{aligned}
$$

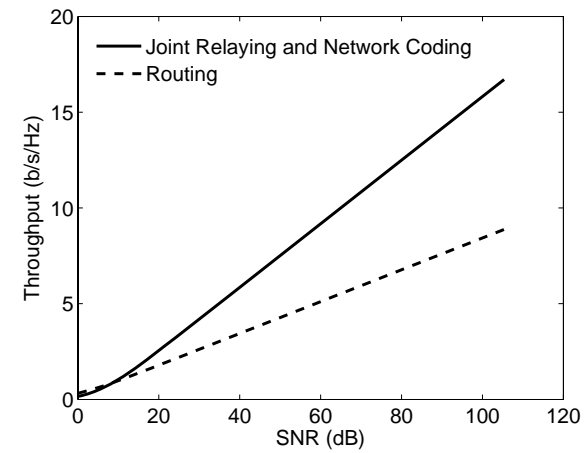

Fig. 2-Network throughput as a function of SNR for half-duplex nodes. At high SNRs, joint relaying and network coding doubles the throughput.

Assuming node 1 knows the link gains $h_{31}$ and $h_{13}$, it cancels its signal out to get

$$
Y_{1}^{\prime}[n]=\alpha h_{31} h_{23} X_{2}[n]+\alpha h_{31} Z_{3}[n]+Z_{1}[n] .
$$

Similarly, the received signal $Y_{2}$ after node 2 cancels its part of the signal is

$$
Y_{2}^{\prime}[n]=\alpha h_{32} h_{13} X_{1}[n]+\alpha h_{32} Z_{3}[n]+Z_{2}[n] .
$$

From (8) and (9), the achievable rates $R_{1}$ and $R_{2}$ can easily be determined to be

$$
\begin{aligned}
& R_{1}=\frac{1}{2} \log \left(1+\frac{\alpha^{2} h_{31}^{2} h_{23}^{2} P}{\alpha^{2} h_{31}^{2}+1}\right) \\
& R_{2}=\frac{1}{2} \log \left(1+\frac{\alpha^{2} h_{32}^{2} h_{13}^{2} P}{A^{2} h_{32}^{2}+1}\right) .
\end{aligned}
$$

In the case the channels are symmetric, we have for $t=$ $1,2 h_{3 t}=h_{t 3}$. We denote $c_{1}=P h_{31}^{2}$ and $c_{2}=P h_{32}^{2}$. The achievable rates (10) and (11) become

$$
\begin{aligned}
& R_{1}=\frac{1}{2} \log \left(1+\frac{c_{1} c_{2}}{2 c_{1}+c_{2}+1}\right) \\
& R_{2}=\frac{1}{2} \log \left(1+\frac{c_{1} c_{2}}{2 c_{2}+c_{1}+1}\right) .
\end{aligned}
$$

\section{Numerical Results}

We next compare the performance of two systems. We plot the overall throughput of the two systems as a function of SNR. For simplicity, we let $c_{1}=c_{2}=c$. From (4), the throughput achievable with routing is bounded by

$$
T=\frac{1}{4} \log (1+2 c)+\frac{1}{4} \log (1+c) .
$$

From (12)-(13), the achieved throughput with the joint encoding scheme is

$$
T^{\prime}=\frac{1}{2} \log \left(1+\frac{c^{2}}{3 c+1}\right) .
$$

Figure 2 shows the throughput of the two schemes as a function of SNR, i.e. channel gain from node 1 to the relay. For high SNR, joint relaying and network coding doubles the throughput since the ratio $T^{\prime} / T \rightarrow 2$ as $\beta \rightarrow \infty$. For low SNR, the throughput of the two systems is comparable and sometimes traditional routing might even be better. For $\beta<$ 


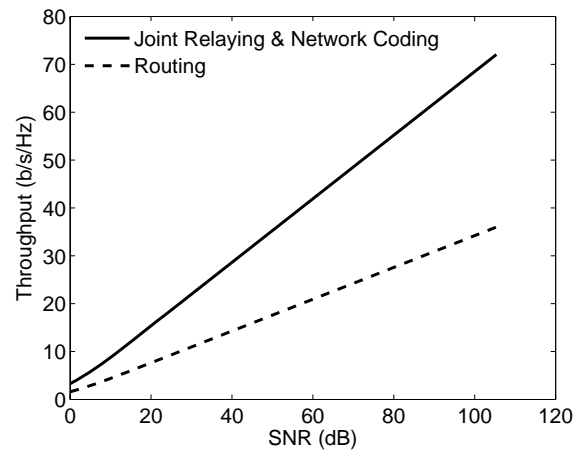

Fig. 3-Network throughput as a function of SNR for the full-duplex case. Joint relaying and network coding provides a higher throughput than routing. At high SNR, it doubles the throughput.

3 , the throughput of the routing scheme is higher. Practical systems typically operate at high SNRs where joint relaying and network coding doubles the throughput.

\section{B. Full-Duplex Relaying}

We next consider the case where nodes can simultaneously send and receive. The analysis follows the one in [7]. For simplicity, we assume symmetric channels.

\section{Routing: The Outer Bound}

Assuming that the two streams fairly share the relay, the routing throughput is bounded by [3]:

$$
\begin{aligned}
C_{1}= & \arg \max _{0 \leq \rho<1} \frac{1}{4} \min \left\{\log \left(1+\left(1-\rho^{2}\right)\left(h_{13}^{2}+h_{12}^{2}\right) P\right)\right. \\
& \left.\log \left(1+h_{12}^{2} P+h_{32}^{2} P+2 \rho \sqrt{h_{12}^{2} h_{32}^{2} P^{2}}\right)\right\} \\
C_{2}= & \arg \max _{0 \leq \rho<1} \frac{1}{4} \min \left\{\log \left(1+\left(1-\rho^{2}\right)\left(h_{23}^{2}+h_{12}^{2}\right) P\right)\right. \\
& \left.\log \left(1+h_{12}^{2} P+h_{31}^{2} P+2 \rho \sqrt{h_{12}^{2} h_{32}^{2} P^{2}}\right)\right\}
\end{aligned}
$$

\section{Network Coding: The Inner Bound}

As in the half-duplex case, the relay amplifies the signal by a factor $\alpha$ given by (6) and broadcasts it. Due to causality, the relay signal is delayed and we have

$$
X_{3}[n]=\alpha Y_{3}[n-1] .
$$

The key difference from the half-duplex case is that nodes 1 and 2 receive each other's signals, combined with the relay signal. The received signals are

$$
\begin{aligned}
Y_{1}^{\prime}[n] & =\alpha h_{31} h_{23} X_{2}[n-1]+h_{21} X_{2}[n] \\
& +\alpha h_{31} Z_{3}[n-1]+Z_{1}[n] \\
Y_{2}^{\prime}[n] & =\alpha h_{31} h_{23} X_{1}[n-1]+h_{12} X_{1}[n] \\
& +\alpha h_{32} Z_{3}[n-1]+Z_{2}[n]
\end{aligned}
$$

The channels at the receivers reduce to two independent Gaussian channels with one tap inter-symbol interference. Using the result of [2], we obtain the rates as in [7] to be

$$
R_{1}=\frac{1}{2} \log \left(1+\frac{k_{1} a+\sqrt{\left(1+k_{1} a\right)^{2}-k_{1}^{2} b^{2}}}{2}\right)
$$

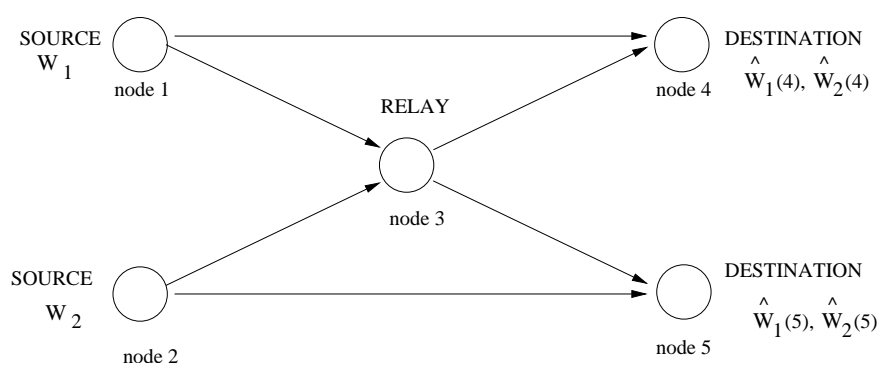

Fig. 4-Multicast relay channel.

$$
R_{2}=\frac{1}{2} \log \left(1+\frac{k_{2} a+\sqrt{\left(1+k_{2} a\right)^{2}-k_{2}^{2} b^{2}}}{2}\right)
$$

where,

$$
\begin{gathered}
k_{1}=\frac{P}{\left(1+\alpha^{2} h_{32}^{2}\right)} \quad k_{2}=\frac{P}{\left(1+\alpha^{2} h_{31}^{2}\right)} \\
a=h_{12}^{2}+\alpha^{2} h_{31}^{2} h_{32}^{2} \quad b=2 \alpha h_{31} h_{32}
\end{gathered}
$$

\section{Numerical Results}

We plot the sum rate of two senders, for both routing and joint encoding. We let $h_{31}=h_{32}=h_{12}$. Figure 3 shows the joint encoding sum rate that is almost double of the routing sum rate. In contrast to the half-duplex case, jointly relaying and coding is always better than routing.

\section{Multicast Relay Channel}

In the previous section we considered the smallest relevant unicast example. We observed that even in that simple network topology, significant throughput gains are obtained through joint relaying and network coding. We now extend these ideas to a more general network to see if similar gains can be obtained. In this section, we expand our network to also analyze the smallest relevant multicast example. We consider a channel model with two senders, two receivers, and a relay, as shown in Fig. 4. The nodes are full-duplex. Encoder $u, u=1,2$, wishes to send a message $W_{u} \in$ $\left\{1, \ldots, M_{u}\right\}$ in $N$ channel uses to its respective decoder $v, v=4,5$ in the unicast case, or to both decoders in the multicast case. We denote the unicast rate from an encoder $u$ to decoder $t$ as $R_{u t}$. The multicast rate from an encoder $u$ is denoted $R_{t}$. Encoders 1 and 2 transmit independent channel inputs $X_{1}$ and $X_{2}$, respectively, and the relay transmits $X_{3}$. The received signal at the relay is given by (1) and signals at the two destination nodes

$$
\begin{aligned}
& Y_{4}=h_{14} X_{1}+h_{24} X_{2}+h_{34} X_{3}+Z_{4} \\
& Y_{5}=h_{15} X_{1}+h_{25} X_{2}+h_{35} X_{3}+Z_{5}
\end{aligned}
$$

where the $Z_{t}$ are independent, zero-mean, unit-variance Gaussian random variables.

\section{A. Routing: The Outer Bound}

During transmission of a block of $N$ bits, the relay timeshares between forwarding information for two encoders, i.e.,

$$
\begin{gathered}
X_{3}[n]=f_{3, n}\left(W_{1}\right) \quad \text { for } 1 \leq n \leq k \\
X_{3}[n]=f_{3, n}\left(W_{2}\right) \quad \text { for } k<n \leq N .
\end{gathered}
$$


Consider the transmission of first $k$ bits. Since we are interested in an outer bound, we can assume that a genie provides $W_{2}$ to nodes 3 and 5 . Then, $X_{2}$ does not interfere with the transmission of the message $W_{1}$. The received signals become

$$
\begin{aligned}
& Y_{3}^{o}=h_{13} X_{1}+Z_{3} \\
& Y_{5}^{o}=h_{15} X_{1}+h_{35} X_{3}+Z_{5}
\end{aligned}
$$

The outer bound on the rate $R_{15}$ achieved in the relay channel specified by (27) and (28) is

$R_{15, k}^{o}=\frac{k}{N} \max _{p\left(x_{1}, x_{3}\right)} \min \left\{I\left(X_{1}, X_{3} ; Y_{5}^{o}\right), I\left(X_{1} ; Y_{3}^{o}, Y_{5}^{o} \mid X_{3}\right)\right\}$

which evaluates to

$R_{15, k}^{o}=\frac{k}{2 N} \max _{0 \leq \rho \leq 1} \min \left\{\log \left(1+P_{1}\left(1-\rho^{2}\right)\left(h_{13}^{2}+h_{15}^{2}\right)\right)\right.$,

$$
\left.\log \left(1+h_{15}^{2} P_{1}+h_{35}^{2} P_{3}+2 \rho h_{15} h_{35} \sqrt{P_{1} P_{3}}\right)\right\} .
$$

We choose $\rho$ such that the two terms in (29) are equal.

The encoder 2 has no choice but to send $W_{2}$ directly to node 4 . For node 4 , both signals $X_{1}$ and $X_{3}$ are interference. We again assume that node 4 obtains $W_{1}$ through a genie, and can cancel interference. Its received signal, (23), becomes

$$
Y_{4}^{o}=h_{24} X_{2}+Z_{4}
$$

achieving the rate

$$
R_{24, k}^{o}=\frac{k}{N} \max _{p\left(x_{2}\right)} I\left(X_{2} ; Y_{4}^{o}\right)
$$

or simply

$$
R_{24, k}^{o}=\frac{k}{2 N} \log \left(1+h_{24}^{2} P_{2}\right) .
$$

For $k<n \leq N$, the roles of the nodes are reversed; node 1 transmits directly achieving the rate:

$$
R_{15, N-k}^{o}=\frac{N-k}{2 N} \log \left(1+h_{15}^{2} P_{1}\right) \text {. }
$$

Relay assists encoder 2 as in (26). For the relay channel:

$$
\begin{aligned}
& Y_{3}^{o}=h_{23} X_{2}+Z_{3} \\
& Y_{4}^{o}=h_{14} X_{1}+h_{34} X_{3}+Z_{4},
\end{aligned}
$$

the relay channel outer bounds equivalent to (29) need to be adapted to reflect the fact that $W_{2}$ was transmitted during $n \leq$ $k$ and so the relay was receiving a priori information about $W_{2}$. We assume that node 3 was able to decode $W_{2}$ during this period. The relay channel upper bound then becomes

$$
\begin{aligned}
R_{24, N-k}^{o}=\frac{N-k}{2 N} & \log \left(1+h_{24}^{2} P_{2}\right. \\
& \left.+h_{34}^{2} P_{3}+2 h_{24} h_{34} \sqrt{P_{2} P_{3}}\right)
\end{aligned}
$$

An outer bound on the unicast rates is thus given by

$$
\begin{aligned}
& R_{15}^{o}=R_{15, k}^{o}+R_{15, N-k}^{o} \\
& R_{24}^{o}=R_{24, k}^{o}+R_{24, N-k}^{o} .
\end{aligned}
$$

Because a genie already provided $W_{1}$ and $W_{2}$ to nodes 4 and 5 respectively, (37)-(38) is also an outer bound on the multicast rates $R_{1}$ and $R_{2}$, just a looser one.

\section{B. Network Coding: An Inner Bound}

We consider a simple scheme that allows the relay to combine two data streams given by

$$
X_{3}[n]=\alpha Y_{3}[n-1],
$$

where $\alpha$ is chosen such that the relay power constraint is satisfied as given by (6). From (23), (24) and (39), the received signal at the destination $t, t=1,2$ at time $n$ becomes

$$
\begin{aligned}
Y_{t}[n] & =h_{1 t} X_{1}[n]+\alpha h_{13} h_{3 t} X_{1}[n-1]+h_{2 t} X_{2}[n] \\
& +\alpha h_{23} h_{3 t} X_{2}[n-1]+\alpha h_{3 t} Z_{3}[n-1]+Z_{t}[n] .
\end{aligned}
$$

Denoting the effective noise as

$$
W_{t}[n]=\alpha h_{3 t} Z_{3}[n-1]+Z_{t}[n]
$$

the received signal (40) becomes

$$
\begin{aligned}
Y_{t}[n] & =h_{1 t} X_{1}[n]+\alpha h_{13} h_{3 t} X_{1}[n-1]+h_{2 t} X_{2}[n] \\
& +\alpha h_{23} h_{3 t} X_{2}[n-1]+W_{t}[n] .
\end{aligned}
$$

The above equation describes a multiaccess (MAC) channel with a unit memory. This observation was also used in [8], [9] to evaluate achievable rates in the multiple-access relay channel. The capacity region of the MAC channel with output $t$ is given by [2, Corollary 1]:

$$
\begin{aligned}
& \mathcal{C}_{\mathrm{MAC}, \mathrm{t}}=\bigcup_{S_{1}(f), S_{2}(f)}\left\{\left(R_{1 t}, R_{2 t}\right): R_{1 t} \geq 0, R_{2 t} \geq 0\right. \\
& R_{1 t} \leq \int_{0}^{1 / 2} \log \left(1+S_{1}(f) T_{1 t}(f)\right) d f \\
& R_{2 t} \leq \int_{0}^{1 / 2} \log \left(1+S_{2}(f) T_{2 t}(f)\right) d f
\end{aligned}
$$

$\left.R_{1 t}+R_{2 t} \leq \int_{0}^{1 / 2} \log \left(1+S_{1}(f) T_{1 t}(f)+S_{2}(f) T_{2 t}(f)\right) d f\right\}$ where

$$
\begin{aligned}
& T_{1 t}(f)=\frac{h_{1 t}^{2}+\alpha^{2} h_{13}^{2} h_{3 t}^{2}+2 \alpha h_{1 t} h_{13} h_{3 t} \cos 2 \pi f}{\alpha^{2} h_{3 t}^{2}+1} \\
& T_{2 t}(f)=\frac{h_{2 t}^{2}+\alpha^{2} h_{23}^{2} h_{3 t}^{2}+2 \alpha h_{2 t} h_{23} h_{3 t} \cos 2 \pi f}{\alpha^{2} h_{3 t}^{2}+1}
\end{aligned}
$$

and $S_{t}(f)$ are the input power spectral densities. The choice of rates $R_{u t}$ that belong to the region (43) for $t=1,2$ will guarantee that $W_{1}, W_{2}$ are multicast to both nodes 4 and 5 . Note that under the assumption that there is no delay at the relay, the outputs (42) describe two MAC channels for which the capacity regions is easily calculated to be

$$
\begin{aligned}
& \mathcal{C}=\left\{\left(R_{1 t}, R_{2 t}\right): R_{1 t} \geq 0, R_{2 t} \geq 0,\right. \\
& R_{1 t} \leq \frac{1}{2} \log \left(1+\frac{P_{1}\left(h_{1 t}+\alpha h_{13} h_{3 t}\right)^{2}}{\left(\alpha h_{3 t}\right)^{2}+1}\right) \\
& R_{2 t} \leq \frac{1}{2} \log \left(1+\frac{P_{2}\left(h_{2 t}+\alpha h_{23} h_{3 t}\right)^{2}}{\left(\alpha h_{3 t}\right)^{2}+1}\right)
\end{aligned}
$$




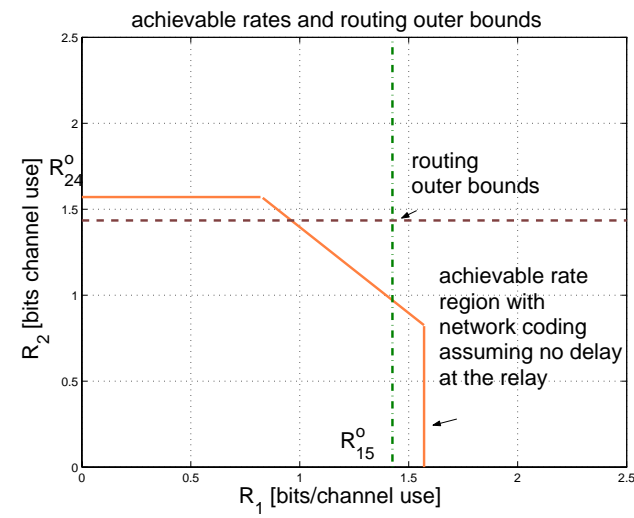

Fig. 5 - Outer bounds on routing and achievable rates with joint relaying and network coding. For all values of $k$, the achievable rate region by network coding is always better than routing.

$$
\begin{aligned}
& R_{1 t}+R_{2 t} \leq \frac{1}{2} \log \\
& \left.\quad\left(1+\frac{P_{1}\left(h_{1 t}+\alpha h_{13} h_{3 t}\right)^{2}+P_{2}\left(h_{2 t}+\alpha h_{23} h_{3 t}\right)^{2}}{\left(\alpha h_{3 t}\right)^{2}+1}\right)\right\}
\end{aligned}
$$

\section{Numerical Results}

We next compare the routing outer bounds to the network coding performance for a particular network scenario. We choose $P_{1}=P_{2}=50, P_{3}=10$. The comparison is shown in Fig. 5. We evaluate the outer bound (37)-(38) for the choice of $k$ for which the two bounds are equal. In this example, to obtain the rates achievable with network coding, we consider the simpler case for which there is no delay at the relay and the rates are in the intersection of two MAC channels given by (46). We observe from Fig. 5 that the achievable rate region contains rates that are not possible with pure relaying. Changing the value of $k$ will increase one of the bounds, but decrease the other one and hence there will always be a set of rates achievable with network coding that outperforms the routing outer bound. However, when the delay is taken into account, the constant power allocation is no longer optimal in (43) and in fact, such choice of powers is not enough to outperform the routing outer bound. We suspect that this is due to the loose relay bounds that do not take the interference into account due to a genie. We are currently working on tightening outer bounds. This will allow for a stronger statement about the network coding gains.

The outer bound (37)-(38) and a relay upper bound [3] for different relay positions, i.e. different values of $y_{3}$, are shown in Fig. 6. Case $y_{3}=2$ corresponds to the scenario of Fig. 4 and hence the routing outer bound corresponds to the bound $R_{15}^{o}$ in Fig. 5. In Fig. 4, the relay upper bound is obtained by assuming that the relay constantly forwards message $W_{1}$. The gap is the penalty due to direct transmission of a message part of the time.

\section{CONCLUSION}

Traditional wireless routing and MAC protocols strive to avoid scheduling multiple data streams at the same time because they interfere with each other, which is considered to

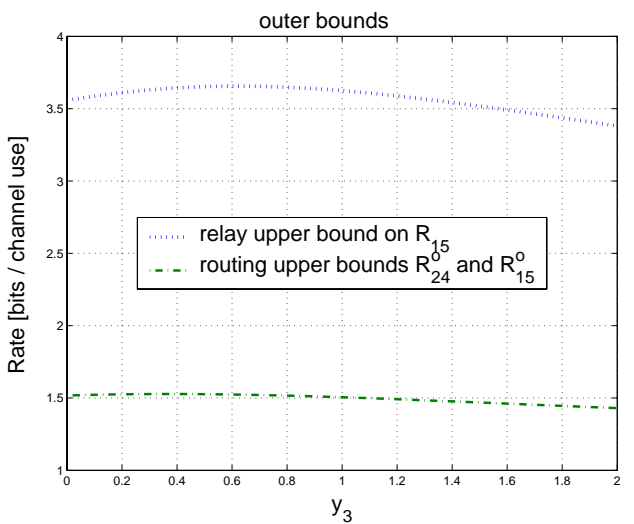

Fig. 6-Outer bounds on routing for different positions of the relay.

be harmful. This paper presents a joint relaying and network code design, where multiple streams are allowed to interfere. The relay forwards the interfering signals creating a form of analog network coding. We show that joint relaying and network coding offers substantial benefits over traditional routing. Furthermore, unlike the more complex relaying strategies, this approach scales well to larger networks and yields non-trivial achievable regions.

Our current work extends the multicast relay channel analysis to more realistic scenarios that take into account the delay at the relay. We are also developing tighter outer bounds to the routing performance to further demonstrate the gains from the joint approach. And while in this paper a simple joint encoding strategy was considered, we plan to investigate various joint encoding schemes, such as extensions of decode-and-forward and compress-and-forward and compare their performance. Subsequent future work will extend this approach to general network scenarios and verify if there are conditions under which separation between network and channel coding might hold.

\section{REFERENCES}

[1] A. B. Carleial. Multiple-access channels with different generalized feedback. IEEE Trans. Inf. Theory, 28(6):841-850, Nov. 1982.

[2] R. Cheng and S. Verdú. Gaussian multiaccess channels with ISI: Capacity region and multiuser water-filling. IEEE Trans. Inf. Theory, 39(3):773-785, May 1993.

[3] T. Cover and A. E. Gamal. Capacity theorems for the relay channel. IEEE Trans. Inf. Theory, 25(5):572-584, Sept. 1979.

[4] G. Kramer, M. Gastpar, and P. Gupta. Cooperative strategies and capacity theorems for relay networks. IEEE Trans. Inf. Theory, 51(9):3037-3063, Sept. 2005.

[5] G. Kramer and A. J. van Wijngaarden. On the white gaussian multipleaccess relay channel. In IEEE Int. Symp. Inf. Theory, June 2000.

[6] E. C. V. D. Meulen. Three-terminal communication channels. Adv. Appl. Prob., 3:120-154, June 1971.

[7] B. Rankov and A. Wittneben. Achievable rate regions for the two-way relay channel. In IEEE Int. Symp. Inf. Theory, July 2006.

[8] L. Sankaranarayanan, G. Kramer, and N. Mandayam. Capacity theorems for the multiple-access relay channel. In Allerton Conference on Communications, Control and Computing, Sept. 2004.

[9] L. Sankaranarayanan, G. Kramer, and N. Mandayam. Hierarchical sensor networks: Capacity theorems and cooperative strategies using the multiple-access relay channel model. In IEEE 1st Annual Conference on Sensor Networks, Oct. 2004.

[10] C. E. Shannon. Two-way communication channels. In Berkeley Symp. Math. Stat. and Prob., volume 1, pages 611-644, 1961. 